\title{
Radiographic, Computed Tomographic and Cellular Phenotypic Features of Primary Nasal Transmissible Venereal Tumors in Four Dogs
}

\section{Muhammad Waseem Aslam ( $\sim$ dr.waseemaslam@gmail.com )}

Faculty of Veterinary Medicine, Universiti Putra Malaysia (UPM), 43400 UPM, Serdang, Selangor, Malaysia. https://orcid.org/0000-0002-4256-5961

\section{Seng Fong Lau ( $\square$ lausengfong@hotmail.com)}

Faculty of Veterinary Medicine, Universiti Putra Malaysia (UPM), 43400 UPM, Serdang, Selangor, Malaysia. https://orcid.org/0000-0003-1283-4355

\section{Puteri Azaziah Megat Abdul Rani}

Faculty of Veterinary Medicine, Universiti Putra Malaysia (UPM), 43400 UPM, Serdang, Selangor, Malaysia.

\section{Ikhwan Saufi Ahamad Azahari}

Faculty of Veterinary Medicine, Universiti Putra Malaysia (UPM), 43400 UPM, Serdang, Selangor, Malaysia.

\section{Case Report}

Keywords: Keywords: Canine transmissible venereal tumor, modified Adams staging, computed tomography, vincristine sulphate, cellular phenotype

Posted Date: August 21st, 2020

DOl: https://doi.org/10.21203/rs.3.rs-62779/v1

License: (c) (i) This work is licensed under a Creative Commons Attribution 4.0 International License. Read Full License 


\section{Abstract}

The authors have removed this preprint from Research Square. 